\title{
An Enhanced Fuzzy Multi Criteria Decision Making Model with a proposed Polygon Fuzzy Number
}

\author{
Samah Bekheet \\ Department of Computer and \\ Information Science, ISSR, Cairo \\ University, Egypt
}

\author{
Ammar Mohammed \\ Department of Computer and \\ Information Science, ISSR, Cairo \\ University, Egypt
}

\author{
Hesham A. Hefny \\ Department of Computer and \\ Information Science, ISSR, Cairo \\ University, Egypt
}

\begin{abstract}
Decisions in real world applications are often made under the presence of conflicting, uncertain, incomplete and imprecise information. Fuzzy multi Criteria Decision making (FMCDM) approach provides a powerful approach for drawing rational decisions under uncertainty given in the form of linguistic values.

Linguistic values are usually represented as fuzzy numbers. Most of researchers adopt either triangle or trapezoidal fuzzy numbers. Since triangle, intervals, and even singleton are special cases of Trapezoidal fuzzy numbers, so, for most researchers' Trapezoidal fuzzy numbers are considered, generalized fuzzy numbers (GFN). In this paper, we introduce polygon fuzzy number (PFN) as the actual form of GFN. The proposed form of PFN provides higher flexibility to decision makers to express their own linguistic rather than other form of fuzzy numbers. The given illustrative example ensures such ability for better handling of the FMCDM problems.
\end{abstract}

Keywords-Fuzzy multi criteria decision making; linguistic values; polygon fuzzy number; level set.

\section{INTRODUCTION}

Decision making is the procedure to find the best alternatives among a set of feasible alternatives and also ranking them as their priorities. Under a fuzzy environment, fuzzy multi criteria decision making (FMCDM) needs linguistic values (e.g. excellent, very good, good, bad) to enable the decision makers to express their own opinions. Such linguistic values need fuzzy tools to evaluate their calculations [1].

Examples of FMCDM tools are T-Norm Based, Gaussian fuzzy numbers, Interval fuzzy numbers, Interval type two fuzzy number, Triangle fuzzy numbers and Trapezoidal fuzzy numbers [2].

Interval, Triangle and Trapezoidal fuzzy numbers are more popular due to their conveniences of the arithmetic operations such as: addition, subtraction, multiplication, division, reciprocal, geometric mean, etc. Such operations enable the decision makers to determine the rank of criteria (alternatives) powerfully [3].

Several researchers consider Trapezoidal fuzzy numbers a s Generalized fuzzy numbers $[4,5,6]$. This mainly due to the fact that other popular forms of specific fuzzy numbers including: triangles, intervals, or even singleton can be obtained as special cases of Trapezoidal fuzzy numbers. The main contribution of this paper is to introduce the polygon fuzzy number as a general fuzzy number rather than the trapezoidal one. Using fuzzy polygon number, several fuzzy numbers can be obtained (e.g. triangle, trapezoidal, pentagon, hexagon, heptagon, octagon and etc).

Such generalization introduces more flexibility to decision makers their own linguistic values. It also to express gives the possibility to standardize different fuzzy numbers into a specific polygon fuzzy number to solve various decision making problems based on different views of decision makers [7, 8, 9].

The rest of the paper is organized as follows: Section II defines the problem. Section III introduces the proposed model and the required definitions of the proposed model. Section IV introduces a numerical example. Finally section V presents the conclusion.

\section{PROBlem Definition}

A Trapezoidal fuzzy number with its four vertices, is considered a generalized number for other forms including: triangles, intervals and also singletons (i.e. crisp numbers) $[4,5,6]$.

However, it is intuitively clear that allowing more vertices to the fuzzy number adds more flexibility to the decision maker to represent his own opinion to deal with the considered FMCDM problem.

Therefore, the ability to introduce generalized piece-wise membership function with n-vertices as a fuzzy number with its own arithmetic operations represents the typical unification of all other forms of fuzzy numbers. Adopting such new forms of generalized fuzzy numbers should considerably enhance modeling and solving FMCDM problems.

\section{Polygon FuZZy Number}

\section{A. Basic Definitions}

Polygon fuzzy sets are firstly addressed in [10] in the context of fuzzy interpolative reasoning. A polygon fuzzy set $A$ has $n$ characteristic p o in ts $\left(\mathrm{a}_{0}, \mathrm{a}_{1}, \ldots, \mathrm{a}_{\mathrm{n}-1}\right)$ as shown in fig. 
1. The core of the fuzzy set, at which the membership equals one, is represented by the interval $\left[\mathrm{a}_{(\mathrm{n}-1 / 2)^{\rfloor}}, \mathrm{a}_{\left.\left[{ }_{[\mathrm{n}}-1 / 2\right)_{]}\right]}\right]$.

There are $L_{(n-1) / 2\rfloor+1}$ membership levels including bottom and top levels. Thus the cardinality of the level set of a polygon fuzzy set is denoted by $\mathrm{V}$ as given in (1)

$$
\mathrm{V}=\mathrm{L}_{(\mathrm{n}-1) / 2\rfloor+1}
$$

It is clear that $\mathrm{V}$ represents the number of $\alpha$-cuts of the polygon fuzzy sets, namely:

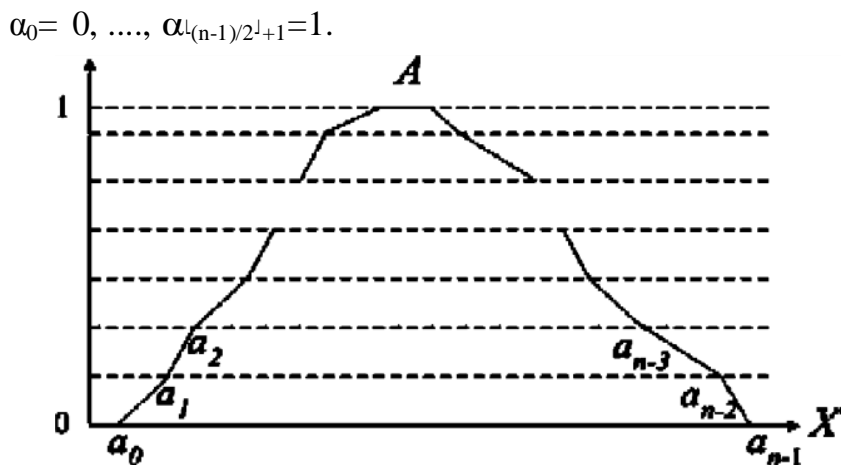

Fig. 1. Polygon fuzzy set

A polygon fuzzy number (PFN) is defined as a convex and normal polygon fuzzy set.

\section{B. Ranking of Polygon fuzzy number}

The centre of area of a fuzzy number is considered the most popular ranking method [11] [12].

Computing the centroid of a general polygon fuzzy number can be obtained by the following theorem.

Theorem 1: Let A be a polygon fuzzy number that is characterized by the $\mathrm{n}$-points $\left(\left(\mathrm{x}_{0}, \mathrm{x}_{1}, \ldots, \mathrm{x}_{\mathrm{n}}\right.\right.$ -

1). Let $y_{i}=\mu_{A}\left(x_{i}\right)$, be the membership level for

each point $\mathrm{x}_{\mathrm{i}}$. Then the centroid $x$ of $\mathrm{A}$ is

Computed using the following formula:

$$
\begin{gathered}
\bar{x}=\frac{\sum_{i=1}^{n} \overline{X_{i}} A_{i}}{\sum_{i=1}^{n} A_{i}} \\
\bar{X}_{i}=\frac{1}{3}\left[\left(x_{i}+x_{i-1}\right)+\left(\frac{\left.\left.x_{i} y_{i}+x_{i-1} y_{i-1}\right)\right]}{y_{i}+y_{i-1}}\right)\right] \\
A_{i}=\frac{\left(x_{i}-x_{i-1}\right)\left(y_{i}+y_{i-1}\right)}{2}
\end{gathered}
$$

\section{Proof:}

The polygon fuzzy number $\mathrm{A}$ with its $\mathrm{n}$ vertices see fig 2, can be divided into ( $\mathrm{n}-1)$ sub-polygons.

Each sub-polygon can generally be represented as a trapezoidal with 4 -vertices. Thus the $\mathrm{i}^{\text {th }}$ sub-polygon as shown in fig.3, has an area equals $A_{i}$ and its centroid $\overline{\boldsymbol{x}_{i}}$ is computed as follows:

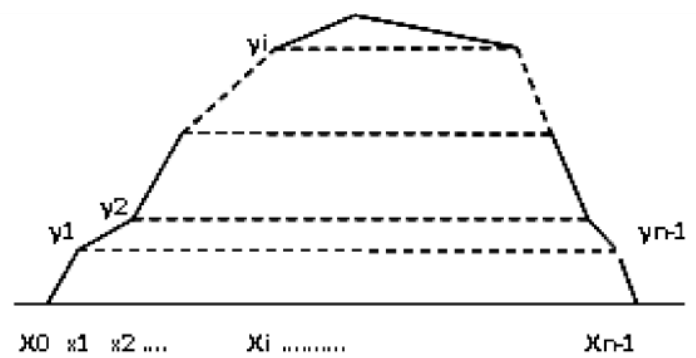

Fig. 2. Polygon characterized

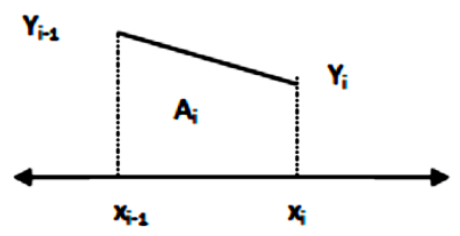

Fig. 3. Sub- polygon area

$$
\begin{gathered}
\overline{x_{i}}=\frac{\int_{\boldsymbol{x}_{i-1}}^{x_{i}} x f_{i}(x) d x}{\int_{\boldsymbol{x}_{i-1}}^{\boldsymbol{x}_{i}} f_{i}(x) d x} \\
=\frac{\boldsymbol{I}_{1}}{\boldsymbol{I} 2}=\frac{f_{i}(x)-y_{i-1}}{x-x_{i-1}}=\frac{y_{i}-y_{i-1}}{x_{i}-x_{i-1}} \\
f_{i}(x)=\frac{y_{i}-y_{i-1}}{x_{i}-x_{i-1}}\left(x-x_{i-1}\right)+y_{i-1} \\
=\frac{y_{i}-y_{i-1}}{x_{i}-\frac{y_{i}-y_{i-1}}{x_{i-1}} x_{i}-x_{i-1}} y_{i-1}+y_{i-1} \\
=\frac{\Delta y_{i}}{\Delta x_{i}}+y_{i-1}-\frac{\Delta y_{i}}{\Delta x_{i}} \mathbf{x}_{i-1}
\end{gathered}
$$

Therefore,

$$
f_{i}(x)=\alpha_{i} x+\beta_{i}
$$

where

$$
\alpha_{i}=\frac{\Delta y_{i}}{\Delta x_{i}}, \beta_{i=} y_{i-1}-\frac{\Delta y_{i}}{\Delta x_{i}} x_{i-1}
$$

Then, performing the integrations for both $I_{1}$ and $I_{2}$, we have:

$\overline{x_{i}}=\frac{1}{3}\left[\left(x_{i}+x_{i-1}\right)+\left(\frac{x_{i} y_{i}+x_{i-1} y_{i-1}}{y_{i}+y_{i-1}}\right)\right]$

Also, it is clear that the area of the $i^{\text {th }}$ sub-polygon shown in fig. 3 is: 


$$
A_{i}=\frac{\left(x_{i}-x_{i-1}\right)\left(y_{i}+y_{i-1}\right)}{2}
$$

\section{Arithmetic Operations}

The arithmetic operations of two polygon fuzzy numbers should satisfy the following two rules:

1) Both fuzzy numbers should have the same number of vertices.

2) Both fuzzy numbers should have the same

level set.

Thus, if we have to add two different polygon fuzzy numbers, e.g triangle $\mathrm{T}\left(\mathrm{a}_{0}, \mathrm{a}_{1}, \mathrm{a}_{2}\right)$ and hexagon $\mathrm{H}\left(\mathrm{b}_{0}, \mathrm{~b}_{1}\right.$, $\left.b_{2}, b_{3}, b_{4}, b_{5}\right)$. If the level set of the triangle is: $\left\{\mathrm{T}\left(\mathrm{a}_{0}\right)=\mathrm{T}\left(\mathrm{a}_{2}\right)=0, \quad\left(\mathrm{a}_{1}\right)=1\right\}$, and the level set of the hexagon is: $\left\{\mathrm{H}\left(\mathrm{b}_{0}\right) \quad=\mathrm{H}\left(\mathrm{b}_{5}\right)=0, \quad \mathrm{H}\left(\mathrm{b}_{1}\right)=\mathrm{H}\left(\mathrm{b}_{4}\right)=0.6\right.$, $\left.\mathrm{H}\left(\mathrm{b}_{2}\right)=\mathrm{H}\left(\mathrm{b}_{3}\right)=1\right\}$.

Then additional level set value at 0.6 should be added to the triangle fuzzy number and consequently more vertices appears for that triangle to be in the form: $\mathrm{T}\left(\mathrm{a}_{0}, \mathrm{a}_{1}, \mathrm{a}_{2}, \mathrm{a}_{3}, \mathrm{a}_{4}\right.$, $\left.\mathrm{a}_{5}\right)$ so that its level set becomes: $\quad\left\{\mathrm{T}\left(\mathrm{a}_{0}\right)=\mathrm{T}\left(\mathrm{a}_{5}\right)=0\right.$, $\left.\mathrm{T}\left(\mathrm{a}_{1}\right)=\mathrm{T}\left(\mathrm{a}_{4}\right)=0.6, \mathrm{~T}\left(\mathrm{a}_{2}\right)=\mathrm{T}\left(\mathrm{a}_{3}\right)=1\right\}$.

Thus, keeping the above tworules in mind,

Assume that there two PFNs $A\left(a_{0}, a_{1}, a_{2}, \ldots ., a_{n-1}\right)$ and $B\left(b_{0}, b_{1}, b_{2}, \ldots ., b_{n-1}\right)$, then the arithmetic operations can be defined as follows:

\section{1- PFNs Addition}

$A \oplus B=\left(a_{0}+b_{0}, a_{1}+b_{1}, a_{2}+b_{2}, \ldots . ., a_{n-1}+b_{n-1}\right)$

\section{2- PFNs Subtraction}

$$
A \ominus B=\left(a_{0}-b_{n-1}, a_{1}-b_{n-2}, a_{2}-b_{n-3}, \ldots . ., a_{n-1}-b_{0}\right)
$$

\section{3- PFNs Multiplication}

$A \otimes B=\left(a_{0} \times b_{0}, a_{1} \times b_{1}, a_{2} \times b_{2}, \ldots . ., a_{n-1} \times b_{n-1}\right)$

4- PFNs Divisions

$$
\text { A } \Phi B=\left(a_{0} / b_{n-1}, a_{1 /} b_{n-2}, a_{2} / b_{n-3}, \ldots . ., a_{n-1} / b_{0}\right)
$$

Where $b_{0} \neq 0, b_{1} \neq 0, \ldots \ldots$. and $b_{n-1} \neq 0$.

\section{ILLUSTRATIVE EXAMPE}

Assume two alternatives are evaluated w.r.t three criteria $\mathrm{C} 1, \mathrm{C} 2$ and $\mathrm{C} 3$ as shown in table 1. The scale of evaluation extends from 1 to 9. Let the decision maker put his evaluation values in the form of polygon fuzzy numbers as shown in table1.

According to table 2, the whole level set is $\{0,0.5,0.6,0.7,0.8,1\}$. Therefore, all the above PFNs should be rewritten according to the whole level set. This of course will add more vertices as shown in table 3 . It is clear, that the obtained unified PFNs are all having twelve vertices at the above six values of the level set.
TABLE I. CRITERIA-AlternAtives EVALUATION MATRIX

\begin{tabular}{|c|c|c|}
\hline Fuzzy number & Type & Level set \\
\hline $\mathrm{A} 11(1,2,3,4)$ & Trapezoid & $\begin{array}{l}\left\{\begin{array}{l}\mathrm{A} 11(1)=\mathrm{A} 11(4)=0, \\
\mathrm{~A} 11(2)\end{array}\right.\end{array}$ \\
\hline A12 $((3,4,5)$ & Triangle & $\begin{array}{l}\left\{\begin{array}{l}\mathrm{A} 12(3)=\mathrm{A} 12(5)=0, \\
\mathrm{~A} 12(4)\end{array}\right.\end{array}$ \\
\hline A13 $(4,5,6,7,8,9)$ & Hexagon & $\begin{array}{l}\{\mathrm{A} 13(4)=\mathrm{A} 13(9)=0, \\
\mathrm{A} 13(5) \\
=0.6, \mathrm{~A} 13(6)=\mathrm{A} 13\end{array}$ \\
\hline $\mathrm{B} 11(1,2,3,4,5)$ & Pentagon & $\begin{array}{l}\{\mathrm{B} 11(1)=\mathrm{B} 11(5)=0, \\
\mathrm{B} 11(2)=0.6, \mathrm{~B} 11(3) \\
=\mathrm{B} 11(4)\end{array}$ \\
\hline $\mathrm{B} 12((2,3,4,6)$ & Trapezoid & $\begin{array}{l}\{\mathrm{B} 12(2)=\mathrm{B} 12(6) \\
=0, \\
\mathrm{D} 12(2)=\mathrm{D} 12(4)=\end{array}$ \\
\hline B $13(4,5,7,8,9)$ & Pentagon & $\begin{array}{l}\{\mathrm{B} 13(4)=\mathrm{B} 13(9)=0, \\
\mathrm{B} 13(5)=\mathrm{B} 13(8)=0.5 \\
\mathrm{~B} 13(7)=1\}\end{array}$ \\
\hline
\end{tabular}

\begin{tabular}{|c|c|c|c|}
\hline Criteria & C1 & C2 & C3 \\
\hline A & $\mathrm{A}_{11}$ & $\mathrm{~A}_{12}$ & $\mathrm{~A}_{13}$ \\
\hline B & $\mathrm{B}_{11}$ & $\mathrm{~B}_{12}$ & $\mathrm{~B}_{13}$ \\
\hline
\end{tabular}

TABLE II. The Evaluation PfNS

Now, get the normalized ranked PFN for each alternative as:

$$
\begin{aligned}
& \mathrm{R}(\mathrm{A})=\sum \mathrm{A}_{1 \mathrm{k}} /\left(\sum \mathrm{A}_{1 \mathrm{k}}+\sum \mathrm{B}_{1 \mathrm{k}}\right), \mathrm{k}=1,2,3 \quad \text { (5) } \mathrm{R}(\mathrm{B})=\sum \\
& \mathrm{B}_{1 \mathrm{k}} /\left(\sum \mathrm{A}_{1 \mathrm{k}}+\sum \mathrm{B}_{1 \mathrm{k}}\right), \mathrm{k}=1,2,3
\end{aligned}
$$

Table 4 shows the obtained normalized ranked PFNs.

TABLE III. THE NEW FormS OF EVALUATION PFNS

\begin{tabular}{|c|c|c|c|c|c|c|}
\hline $\begin{array}{c}\text { PFN } \\
\text { level }\end{array}$ & $\mathrm{A}_{11}$ & $\mathrm{~A}_{12}$ & $\mathrm{~A}_{13}$ & $\mathrm{~B}_{11}$ & $\mathrm{~B}_{12}$ & $\mathrm{~B}_{13}$ \\
\hline 0 & 1 & 3 & 4 & 1 & 2 & 4 \\
\hline 0.5 & 1.62 & 3.5 & 4.83 & 1.83 & 2.5 & 5 \\
\hline 0.6 & 1.75 & 3.6 & 5 & 2 & 2.6 & 5.4 \\
\hline 0.7 & 1.88 & 3.7 & 5.25 & 2.25 & 2.7 & 5.8 \\
\hline 0.8 & 2 & 3.8 & 5.5 & 2.5 & 2.8 & 6.2 \\
\hline 1 & 3 & 4 & 6 & 3 & 3 & 7 \\
\hline 1 & 3 & 4 & 7 & 4 & 4 & 7 \\
\hline 0.8 & 3.2 & 4.2 & 7.67 & 4.2 & 4.4 & 7.4 \\
\hline 0.7 & 3.3 & 4.3 & 8 & 4.3 & 4.6 & 7.6 \\
\hline 0.6 & 3.4 & 4.4 & 8.14 & 4.4 & 4.8 & 7.8 \\
\hline 0.5 & 3.5 & 4.5 & 8.29 & 4.5 & 5 & 8 \\
\hline 0 & 4 & 5 & 9 & 5 & 6 & 9 \\
\hline
\end{tabular}


TABLE IV. THE NORMALIZED RANKING PFNS

\begin{tabular}{|c|c|c|}
\hline Normalized & & \\
\hline & & \\
Ranked & & $\mathrm{R}(\mathrm{B})$ \\
\hline 0 & & \\
\hline 0.5 & 0.21 & 0.18 \\
\hline 0.6 & 0.295 & 0.276 \\
\hline 0.7 & 0.316 & 0.3 \\
\hline 0.8 & 0.337 & 0.335 \\
\hline 1 & 0.363 & 0.37 \\
\hline 1 & 0.448 & 0.445 \\
\hline 0.8 & 0.538 & 0.58 \\
\hline 0.7 & 0.66 & 0.7 \\
\hline 0.6 & 0.723 & 0.765 \\
\hline 0.5 & 0.783 & 0.83 \\
\hline 0 & 0.845 & 0.91 \\
\hline & 1.2 & 1.33 \\
\hline
\end{tabular}

Now, applying the above centroid theorem for PFN, then the final crisp rank value for each alternative is found as follows:

$\operatorname{COA}(\mathrm{R}(\mathrm{A}))=0.5244$,

$\operatorname{COA}(\mathrm{R}(\mathrm{B}))=0.468$.

Thus it is clear that alternative A should be selected as the best choice.

\section{CONCLUSION}

This paper introduced a way for adopting PFNs in FMCDM problems. The proposed forms of PFN ensure its generality over other popular forms of fuzzy numbers. A general formula for ranking PFNs is given using the centroid method.

The arithmetic operations for PFNs are clearly illustrated and an illustrative example shows how to adopt such generalized fuzzy numbers for solving FMCDM problems.

\section{REFERENCES}

[1] Dug Hun Hong, Strong laws of large numbers for t-norm-based addition of fuzzy set-valued random variables, Fuzzy sets and systems,(2013) vol. 223, pp 449-728.

[2] Shyi-Ming Chen ,Cheng-Yi Wang, Fuzzy decision making systems based on interval type-2 fuzzy sets, Science Direct, Applied Mathematical Modeling, (2013).

[3] F. Herrera, E. Herrera-Viedma, Spain Linguistic decision analysis: steps for solving decision problems under linguistic information, Computer Science and Arti cial Intelligence (2000) 115, pp 67-82.

[4] Shi-Jay Chen and Shyi-Ming Chen, Fuzzy Risk Analysis Based on Similarity Measures of Generalized Fuzzy Numbers, IEEE Transaction on Fuzzy systems (2003), Vol. 11, No. 1, pp. 45-56.

[5] Dong Yong, Shi Wenkang, Du Feng, Liu Qi, A new similarity measure of generalized fuzzy numbers and its application to pattern recognition, Pattern Recognition Letters, 25, (2004), pp. 875-883.

[6] Shyi-Ming Chen, Jim-Ho Chen, Fuzzy risk analysis based on ranking generalized fuzzy numbers with different heights and different spreads, Expert Systems with Applications, 36, (2009), pp. 6833-6842.

[7] Chia-Chi Sun, A performance evaluation model by integrating fuzzy AHP and fuzzy TOPSIS methods, Expert Systems with Applications 37 (2010) 7745-7754.

[8] Mohammad Anisseh, Fatemeh Piri , Mohammad Reza Shahraki ,Fazlollah Agamohamadi, Fuzzy extension of TOPSIS model for group decision making under multiple criteria, Springer 2011.

[9] E. Roghanian, J. Rahimi, A. Ansari, Comparison of first aggregation and last aggregation in fuzzy group TOPSIS, Applied Mathematical Modelling 34 (2010) 3754-3766.

[10] Yu-Chuan Chang, Shyi-Ming Chen, Churn-Jung Liau, Fuzzy Interpolative Reasoning for Sparse Fuzzy-Rule-Based Systems Based on the Areas of Fuzzy Sets, IEEE Transaction on Fuzzy Systems, Vol. 16, No. 5, pp. 1285-1301, October 2008.

[11] Cheng, C. H., A new approach for ranking fuzzy numbers by distance method, Fuzzy Sets and Systems (1998). 95, 307-317.

[12] Wang, Y.M., Yang, J.B., Xu, D.L., and Chin, K.S., On the centroids of fuzzy numbers, Fuzzy Sets and Systems (2006) 157, pp: 919-926 